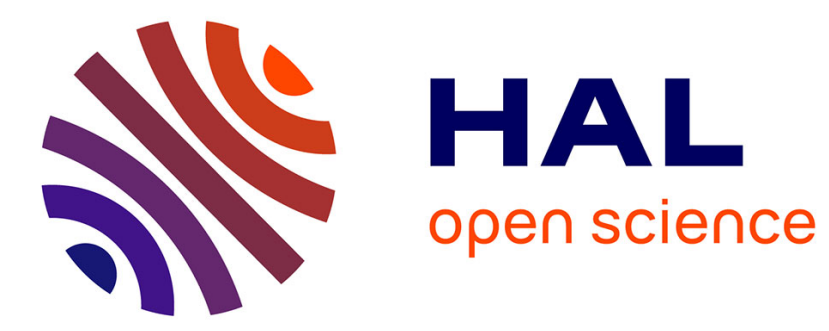

\title{
Falling jets of particles in viscous fluids
}

Florent Pignatel, Maxime Nicolas, Elisabeth Guazzelli, David Saintillan

\section{To cite this version:}

Florent Pignatel, Maxime Nicolas, Elisabeth Guazzelli, David Saintillan. Falling jets of particles in viscous fluids. Physics of Fluids, 2009, 21, pp.123303 - 123303. 10.1063/1.3276235 . hal-01440839

\section{HAL Id: hal-01440839 \\ https://hal.science/hal-01440839}

Submitted on 19 Jan 2017

HAL is a multi-disciplinary open access archive for the deposit and dissemination of scientific research documents, whether they are published or not. The documents may come from teaching and research institutions in France or abroad, or from public or private research centers.
L'archive ouverte pluridisciplinaire HAL, est destinée au dépôt et à la diffusion de documents scientifiques de niveau recherche, publiés ou non, émanant des établissements d'enseignement et de recherche français ou étrangers, des laboratoires publics ou privés. 


\title{
Falling jets of particles in viscous fluids
}

\author{
Florent Pignatel, ${ }^{1}$ Maxime Nicolas, ${ }^{1}$ Élisabeth Guazzelli, ${ }^{1}$ and David Saintillan ${ }^{2}$ \\ ${ }^{1}$ IUSTI CNRS UMR 6595-Polytech' Marseille-Aix-Marseille Université (U1), \\ Technopôle Château-Gombert, 13453 Marseille Cedex 13, France \\ ${ }^{2}$ Department of Mechanical Science and Engineering, University of Illinois at Urbana-Champaign, \\ Urbana, Illinois 61801, USA
}

(Received 24 July 2009; accepted 1 December 2009; published online 18 December 2009)

\begin{abstract}
We have investigated the time evolution of a jet of non-Brownian particles falling under the action of gravity in a viscous liquid at low Reynolds number. Different regimes have been observed depending on volume fraction and particle-to-jet diameter ratio. In particular, the jet has been found to be unstable and to develop varicose modulation of its diameter at low volume fractions. The dominant wavelength and saturated amplitude have been measured and are observed to decrease with increasing volume fraction. A simple numerical simulation using point particles is able to capture the major features of the instability. (C) 2009 American Institute of Physics.
\end{abstract}

[doi:10.1063/1.3276235]

\section{INTRODUCTION}

A vertically falling stream of liquid with a circular crosssection of diameter $D_{j}$ is known to break up into drops. This classical Rayleigh-Plateau instability, by which an axisymmetric varicose perturbation grows in time and eventually leads to drop formation, occurs for an incompressible inviscid liquid having a free surface governed by surface tension. ${ }^{1,2}$ The instability can be shown to occur if the length of the jet exceeds $\pi D_{j}$, with a wavelength of greatest instability $\approx 4.5 D_{j}$. This instability has received much attention since the work of Rayleigh and Plateau, with interesting applications in ink-jet technology. The viscous case of a buoyant-fluid jet is of particular relevance for geophysical flows such as viscous gravity currents and lava flows. It has been considered by Lister ${ }^{3}$ who showed that the flow is linearly unstable in the limit of long wavelength as well as at low but still finite Reynolds number for vanishing surface tension. The viscous-potential-flow analysis of a liquid jet into a gas or another liquid was undertaken more recently by Funada et $a l .{ }^{4}$ This stability analysis, which includes inertial, viscous, and capillary effects, clearly shows that the jet is stable when the Reynolds and Weber numbers tend toward zero.

In the present study, we focus on particulate jets in which the background fluid is identical to the suspending fluid of the suspension. These particulate jets are relevant to various phenomena found in nature and in industry, such as gravity and turbidity currents, the dispersion of pollutants in lakes and oceans, and mixing processes in fluidization reactors. Experiments on gravity-driven suspension jets were performed by Nicolas ${ }^{5}$ for a variety of flow parameters: the jets were created by letting a dense particulate suspension $(\approx 60 \%$ volume fraction) settle by gravity out of a tube into a tank containing the same liquid as that used to prepare the suspension. Depending on the importance of particle and fluid inertia, different jetting regimes were observed, ranging from unstable jets showing varicose perturbations (leading to blob formation) to atomized jets. While these regimes are reminiscent of the behavior of inviscid jets of immiscible liquids, particulate jets do not present surface tension, and therefore the detailed mechanism for instability has yet to be found.

While the experiments of Nicolas focused on dense inertial particulate jets, one might wonder whether the instability persists at low Reynolds number or with more dilute jets. This open question is precisely the topic of present study, which investigates the dynamics of falling jets of particles in a highly viscous fluid, i.e., in creeping flow conditions. Unlike the Nicolas experiments, this study is not restricted to dense suspensions but considers a range of volume fractions from very dilute to concentrated. Using both experiments and numerical simulations based on a point-particle approximation (which are strictly at zero Reynolds number), we observe a varicose instability with axisymmetric modulation of the jet diameter, which never leads to breakup and drop formation, in stark contrast with the inertial case.

A related problem is the sedimentation of a cloud of particles in a viscous fluid. Similarly, an instability is observed in that case even at very low Reynolds number. This instability, however, does result in the breakup of the cloud. Specifically, an initially spherical cloud evolves into a torus, which then becomes unstable and breaks up into smaller droplets, which themselves can become unstable again. An important feature of the dynamics is that the particles circulate in a toroidal vortex inside the cloud and occasionally escape along a vertical tail emanating from its rear. This depletes the region along the vertical axis of the cloud and leads to the torus formation. Numerical simulations using the same point-particle approach as used in the present paper capture these dynamics with a quantitative agreement in the dilute regime. ${ }^{6-8}$

In both the particulate jet and cloud problems, the suspension may be modeled as an effective medium of excess mass. However, the discrete nature of the suspension is fundamental in the understanding of these instabilities. Indeed, the chaotic velocity fluctuations resulting from hydrody- 


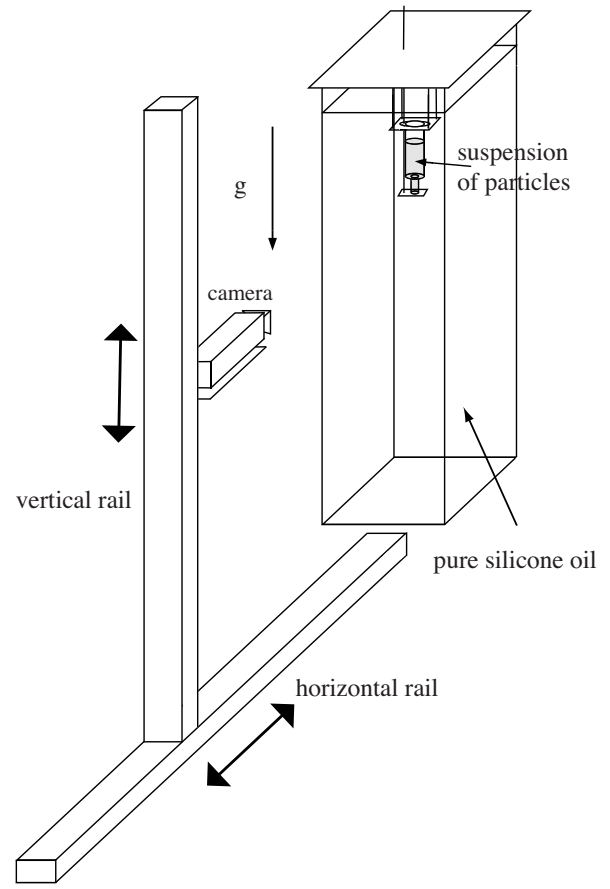

FIG. 1. View of the experimental setup.

namic interactions cause the particle motions to depart from the streamlines predicted by the continuum models. This feature is central in explaining the instability in the case of a cloud, and we speculate that it also plays a role in the jet instability at low Reynolds number. This point is supported by our experiments and simulations as described below.

The experimental setup is described in Sec. II, and the simulation method is described in Sec. III. The data analysis for both experiments and simulations is explained in Sec. IV. A simple continuum model is presented in Sec. V. The general behavior of the jet dynamics is presented in Sec. VI, showing that the instability saturates. This saturation regime is analyzed in detail in Sec. VII, where we compare results from experiments and simulations as well as the predictions of the continuum model. This continuum model provides average quantities of the jet such as the mean diameter and velocity, whereas the point-particle simulations capture the features of the instability such as the fluctuations in jet diameter and the most unstable wavelength. Note again that the continuum models of Lister ${ }^{3}$ and Funada et al. ${ }^{4}$ do not predict instability for vanishing surface tension and Reynolds number. Conclusions are drawn in Sec. VIII, and a qualitative mechanism for the instability is proposed.

\section{EXPERIMENTAL SETUP}

Experiments are performed in a glass-walled tank having an inner cross-section of $20 \times 20 \mathrm{~cm}^{2}$ and a filled height of $\approx 80 \mathrm{~cm}$ as sketched in Fig. 1. All the experiments are performed at the ambient temperature of $22 \pm 2{ }^{\circ} \mathrm{C}$. The fluid used is silicone oil 47V1000 having viscosity $\eta_{f}=1.05 \pm 0.06 \mathrm{~Pa} \mathrm{~s}$ and density $\rho_{f}=972 \pm 19 \mathrm{~kg} \mathrm{~m}^{-3}$. Two batches of glass spheres are used in the experiments (see Table I). The suspension is prepared in a separate small container having curved walls. A slow rotation of the container
TABLE I. Particle characteristics.

\begin{tabular}{lcc}
\hline \hline Batch & $\begin{array}{c}d_{p} \\
(\mu \mathrm{m})\end{array}$ & $\begin{array}{c}\rho_{p} \\
\left(\mathrm{~kg} \mathrm{~m}^{-3}\right)\end{array}$ \\
\hline A & $319 \pm 25$ & $2470 \pm 20$ \\
B & $149 \pm 12$ & $2480 \pm 50$ \\
\hline \hline
\end{tabular}

leads to an efficient mixing of the particles with the fluid without introducing air bubbles. Once prepared at the desired particle volume fraction $\phi_{0}$, the suspension is poured into a vertical cylindrical container, which is then slowly lowered and immersed in the tank. This container has a tube outlet of inner diameter $D_{t}$, which is closed by a gate. Once the gate is opened, the suspension flows down under the effect of gravity, and a vertical suspension jet is formed. The experimental conditions are summarized in Table II. Note that two combinations (sets 1 and 3 ) of outlets having different inner diameters $D_{t}$ and batches of spheres are used to obtain two different experiments having same $D_{t} / d_{p}$. Both the jet Reynolds number, $\operatorname{Re}=\rho_{f} V_{j} D_{t} / \eta_{f}$ (where $V_{j}$ is the jet average velocity) and the particle Reynolds number, $R e_{p}=\operatorname{Re} d_{p} / D_{t}$, are $\ll 1$.

The tank is lit with two side neon lights, which produce a light-sheet of thickness $\approx 2 \mathrm{~cm}$ illuminating the suspension jet. The jet is imaged within this sheet by a 12 frame/s 8 bit digital camera facing the tank and mounted onto a vertical translating stage attached to a horizontal rail. The thickness of the light-sheet is such that the jet is fully illuminated and the images generated therefore show a horizontal projection of the entire jet. Two combinations of cameras and lenses are used. A Basler A102f camera (with a resolution of 1392 $\times 1040$ pixels) having a $55 \mathrm{~mm}$ lens (Nikon micro-Nikkor) records the close evolution of the jet within an imaging window of height $\approx 10 D_{t}$, which can be translated vertically by moving the camera. A Basler A101f camera (with a resolution $1300 \times 1030$ pixels) having a wide angle lens (Nikon AF Nikkor $14 \mathrm{~mm}$ ) records the evolution of the jet within a fixed imaging window of height $\approx 50 D_{t}$. The top of this window is positioned at $\approx 50 D_{t}$ below the bottom of the outlet.

\section{NUMERICAL METHOD}

We simulate a falling jet of particles in an unbounded fluid at rest at infinity using a point-particle approximation in the limit of zero Reynolds number. Particles are injected continuously in time at the top of the jet using a procedure described below and exert a point force on the fluid equal to their buoyant weight $\mathbf{F}$. Denoting by $\mathbf{r}_{i}$ the position of particle $i$, the fluid velocity $\mathbf{u}$ at position $\mathbf{r}$ satisfies the Stokes equations

TABLE II. Experimental conditions.

\begin{tabular}{ccccccc}
\hline \hline Set & $\begin{array}{c}D_{t} \\
(\mathrm{~mm})\end{array}$ & Batch & $\begin{array}{c}\phi_{0} \\
(\%)\end{array}$ & $\begin{array}{c}\ell=\left(d_{p} / 2\right) \phi_{0}^{-1 / 3} \\
(\mu \mathrm{m})\end{array}$ & $\operatorname{Re} \times 10^{2}$ & $\operatorname{Re}_{p} \times 10^{4}$ \\
\hline 1 & 7 & $\mathrm{~A}$ & $2-50$ & $200-588$ & $1-10$ & $5-50$ \\
2 & 7 & $\mathrm{~B}$ & $0.5-50$ & $94-436$ & $0.4-10$ & $0.8-20$ \\
3 & 3.3 & $\mathrm{~B}$ & $2-40$ & $100-274$ & $0.2-1$ & $0.9-6$ \\
\hline \hline
\end{tabular}



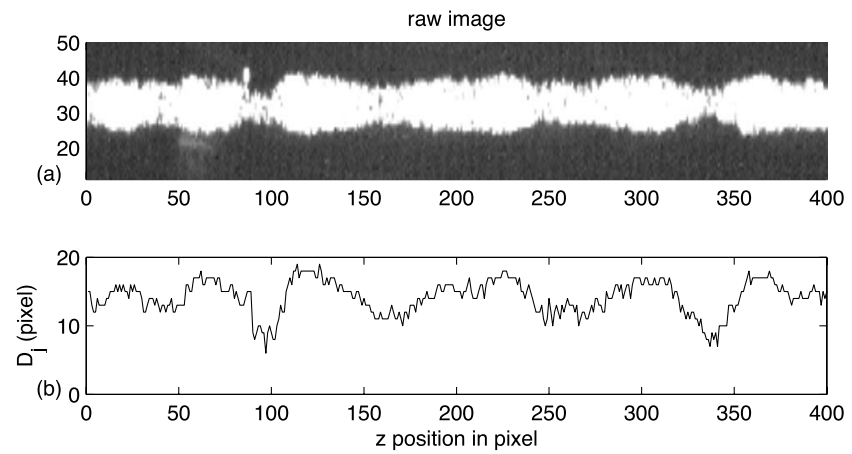

(c)

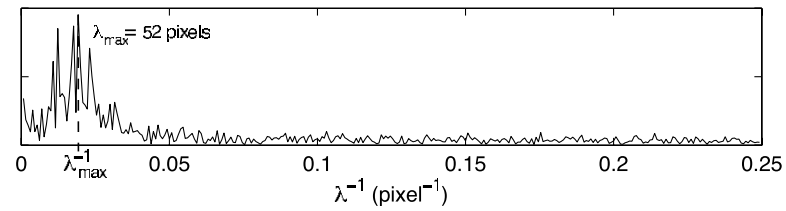

FIG. 2. Method for the determination of the mean jet diameter, standard deviation, and dominant wavelength of the instability. (a) Raw image of a jet in the saturated region (coordinates are in pixels). (b) Jet diameter $D_{j}(z)$ inferred by image analysis of (a). (c) Spatial Fourier transform of $D_{j}(z)$ in which the peak corresponds to the dominant wavelength of the instability.

$$
\eta_{f} \nabla^{2} \mathbf{u}-\nabla p+\sum_{i=1}^{N_{p}(t)} \mathbf{F} \delta\left(\mathbf{r}-\mathbf{r}_{i}\right)=0, \quad \nabla \cdot \mathbf{u}=0
$$

where $p$ is the fluid pressure and $N_{p}(t)$ is the number of particles inside the jet at time $t$. Following the point-particle approach used by Metzger et al., ${ }^{8}$ the particle velocities are then obtained as the sum of the Stokes velocity and of the disturbance fluid velocity induced by the other particles,

$$
\dot{\mathbf{r}}_{i}=\frac{\mathbf{F}}{3 \pi \eta_{f} d_{p}}+\mathbf{F} \cdot \sum_{j \neq i} \mathbf{T}\left(\mathbf{r}_{i j}\right)
$$

where $\mathbf{r}_{i j}=\mathbf{r}_{i}-\mathbf{r}_{j}$ and $\mathbf{T}$ denotes the Oseen-Burgers tensor,

$$
\mathbf{T}(\mathbf{r})=\frac{1}{8 \pi \eta_{f}}\left(\frac{\mathbf{I}}{r}+\frac{\mathbf{r r}}{r^{3}}\right),
$$

where $\mathbf{I}$ is the identity tensor and $r=|\mathbf{r}|$.

Several length scales are available for nondimensionalization of the equations, including the size of the injection region (corresponding to the tube outlet diameter $D_{t}$ in the experiments), the developed jet diameter $D_{j}$, the particle diameter $d_{p}$, and the mean interparticle distance $\ell=\left(d_{p} / 2\right) \phi_{0}^{-1 / 3}$. For the purpose of making the equations dimensionless, we choose the radius $D_{t} / 2$ of the injection region together with the Stokes velocity $F / 3 \pi \eta_{f} d_{p}$ of the particles; this choice will be further discussed below when we present results from the experiments and simulations. The equations of motion (2) for the particles then become

$$
\dot{\mathbf{r}}_{i}=\hat{\mathbf{z}}+\frac{3 d_{p}}{4 D_{t}} \sum_{j \neq i}\left(\frac{\mathbf{I}}{r_{i j}}+\frac{\mathbf{r}_{i j} \mathbf{r}_{i j}}{r_{i j}^{3}}\right) \cdot \hat{\mathbf{z}},
$$

where $\hat{\mathbf{z}}$ is a unit vector pointing in the direction of gravity. Equation (4) is integrated numerically using a second-order Adams-Bashforth time-marching scheme (together with explicit Euler for the first time step), and a time step of $\Delta t=10^{-3}$ is used in all simulations. This choice ensures that

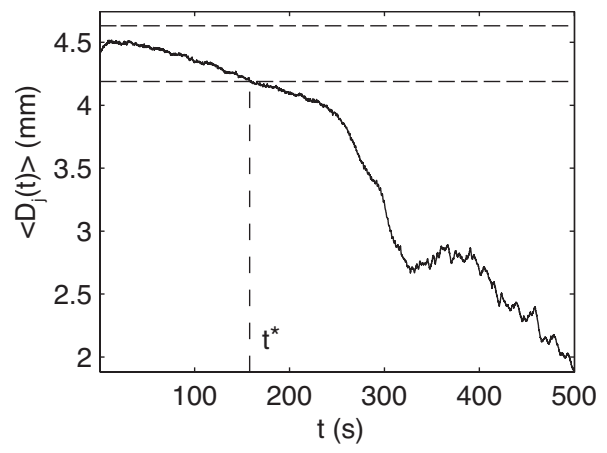

FIG. 3. Time evolution of the mean diameter in a typical experiment using set 1 at $\phi_{0}=21.5 \%$.

the particles only travel a fraction of the mean interparticle distance during each time step. Because of the chaotic nature of the equations of motion, we also find that using smaller time steps produces no significant differences in the dynamics (see Metzger et al. ${ }^{8}$ for a more detailed discussion).

The particle injection procedure consists in introducing particles at each time step at the top of the jet inside an injection region of diameter $D_{t}$ and height $V_{j} \Delta t$, where $V_{j}$ is the local vertical jet velocity. The number of particles introduced and their positions are random but chosen to achieve a desired particle volume fraction on average. Note that even though the particles in the simulations effectively have no volume owing the point-particle approximation, we can still define a volume fraction based on the particle diameter $d_{p}$ appearing in the Stokes velocity. To capture the correct dynamics, it is crucial to choose the injection velocity to match the unknown local jet velocity $V_{j}$. Because $V_{j}$ depends on the number of injected particles, an iteration procedure is required. The number of particles to be injected at a given time step is adjusted until matching is achieved. The particles are then introduced in the jet, and we proceed to the next time step. This procedure can be verified to produce jets with the desired volume fraction.

\section{DATA ANALYSIS}

The experimental images of the suspension jet are postprocessed with MATLAB to obtain the jet diameter $D_{j}(z, t)$, where $z$ is the vertical direction and $t$ is time [see Figs. 2(a) and 2(b)]. First, we subtract from these images a background image taken before the arrival of the jet in the same window with the same settings. Second, the jet images are smoothed by replacing each pixel intensity by the average intensity of its close neighbors. Third, we threshold the gray-level intensity of the resulting images. Finally, the jet boundaries are determined, and the jet diameter $D_{j}(z, t)$ at different heights $z$ and times $t$ is measured. The starting time of the measurement corresponds to a jet extending across the entire height of the window.

Mean $\left\langle D_{j}(t)\right\rangle$ and standard deviation $\sigma_{D_{j}}(t)$ of the diameter values along $z$ are computed at each given time $t$. Figure 3 shows the mean diameter $\left\langle D_{j}(t)\right\rangle$ versus time $t$. This diameter $\left\langle D_{j}(t)\right\rangle$ slowly decreases with time as the injection conditions also evolve with time due to stratification effects and 


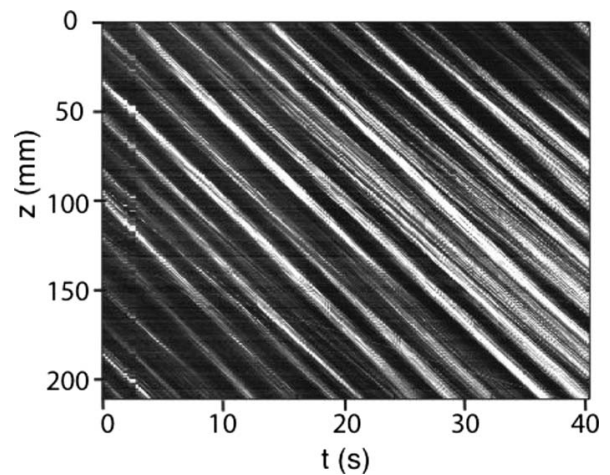

FIG. 4. Spatiotemporal diagram. Vertical axis is positioned along the jet axis. Horizontal axis is time.

influence of the interface between the suspension and clear fluid in the injection container. To measure steady quantities, we consider the time span for which $\left\langle D_{j}(t)\right\rangle$ stays within 5\% of the initial diameter $\left\langle D_{j}(0)\right\rangle$. We then compute the diameter $D_{j}$ averaged over this time span $\left[0, t^{*}\right]$ and its uncertainty, defined as the standard deviation of $\left\langle D_{j}(t)\right\rangle$ over the same time span. In the same way, we obtain $\sigma_{D_{i}}$, the average of $\sigma_{D_{j}}(t)$ over the same time span $\left[0, t^{*}\right]$, and its uncertainty.

The method used for determining the dominant wavelength $\lambda$ of the instability is illustrated in Fig. 2(c). The spatial discrete Fourier transform of the jet diameter along $z$ at a given time $t$ is calculated and exhibits a clear peak corresponding to the dominant wavelength $\lambda(t)$ for the fluctuations in diameter. This quantity is then averaged over the same time span $\left[0, t^{*}\right]$ to yield the mean wavelength $\lambda$ and its error bar. Note that measurements of $\lambda$ are only performed in windows located far downstream from the injection region to ensure that the instability is fully developed.

Spatiotemporal diagrams can directly be obtained from the raw experimental images from which the background image has been subtracted by plotting a vertical line of pixels versus time, as seen in Fig. 4. The measurement of the slope of isointensities gives the velocity of the jet varicose instability $V_{j}(t)$. Again we obtain $V_{j}$ by averaging over the time span $\left[0, t^{*}\right]$. Note that for all averaged quantities over time and space, we drop the brackets for simplicity.

The numerical simulations compute particle positions and velocities versus time. These data are also postprocessed with MATLAB to obtain the jet diameter $D_{j}(z, t)$. In fact, $D_{j}(z, t)$ is computed as the horizontal distance between the two farthest particles in a vertical jet thickness. This thickness is chosen to be not too small to contain enough particles and not too large to avoid smoothing variations. To ensure meaningful comparisons, the same interrogation techniques are used as in the experiments to yield the quantities $D_{j}, \sigma_{D_{j}}$, $\lambda$, and their uncertainties. The only difference lies in the choice of the time span. Since there is no variation in the numerical injection conditions, $\left\langle D_{j}(t)\right\rangle$ does not decrease with time as in the experiments but experiences fluctuations due to the particle interactions. We therefore consider a time span for which $\left\langle D_{j}(t)\right\rangle$ remains constant with time within $0.1 \%$.
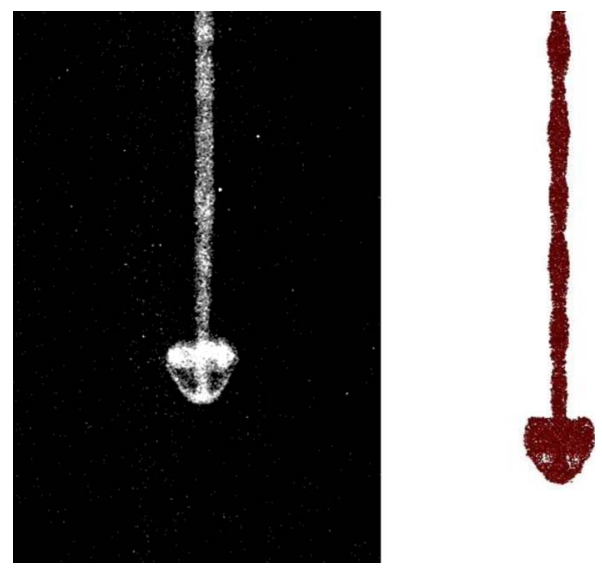

FIG. 5. (Color online) Snapshots of the jet instability at $\phi_{0}=5 \%$ in the experiment (left) and simulation (right) (enhanced online). [URL: http://dx.doi.org/10.1063/1.3276235.1]

Unlike in the experiments, the numerical simulations directly provide the velocity of the particles. The jet velocity is computed by considering the velocity average over the same thickness used in the computation of the jet diameter $D_{j}(z, t)$ described above. The velocities obtained by this latter method can be shown to be slightly lower than those obtained using spatiotemporal diagrams, although the measured differences typically remain within error bars.

\section{CONTINUUM MODEL}

We consider a particulate fluid modeled as a continuum having a density $\rho_{j}=(1-\phi) \rho_{f}+\phi \rho_{p}$ and an effective viscosity modeled by Krieger empirical relation ${ }^{9} \eta_{j}=\eta_{f} / \eta^{*}$ $=\eta_{f}\left(1-\phi / \phi_{c}\right)^{-1.68}$, where $\phi_{c}=0.64$ is the close-packing volume fraction. This effective fluid is released out of a cylindrical tube (radius $D_{t} / 2$ ) into a cylindrical container (radius $D_{c} / 2$ ) filled with pure fluid at rest having density $\rho_{f}$ and viscosity $\eta_{f}$. The flow is driven by the density difference between the suspension and the pure fluid and is characterized by a buoyancy number $N_{b}=\left(\rho_{j}-\rho_{f}\right) g D_{t}^{2} / 4 \eta_{j} V_{t}$ comparing the effects of gravitational and viscous forces. The flow is assumed to be steady, parallel, and axisymmetric, i.e., the velocities are only considered along $z$ and only depend on the radial coordinate $r$.

Under these assumptions, the continuum model is written as four coupled equations. The first one is a Stokes equation for the suspension flowing inside the tube,

$$
\eta_{j} \frac{1}{r} \frac{\partial}{\partial r}\left(r \frac{\partial v_{t}}{\partial r}\right)+\left(\rho_{j}-\rho_{f}\right) g-K=0,
$$

where $K$ is an effective pressure gradient. The second equation of the model is a Stokes equation for the jet velocity $v_{j}(r)$ below the outlet of the tube, driven by gravity,

$$
\eta_{j} \frac{1}{r} \frac{\partial}{\partial r}\left(r \frac{\partial v_{j}}{\partial r}\right)+\left(\rho_{j}-\rho_{f}\right) g-K=0 .
$$



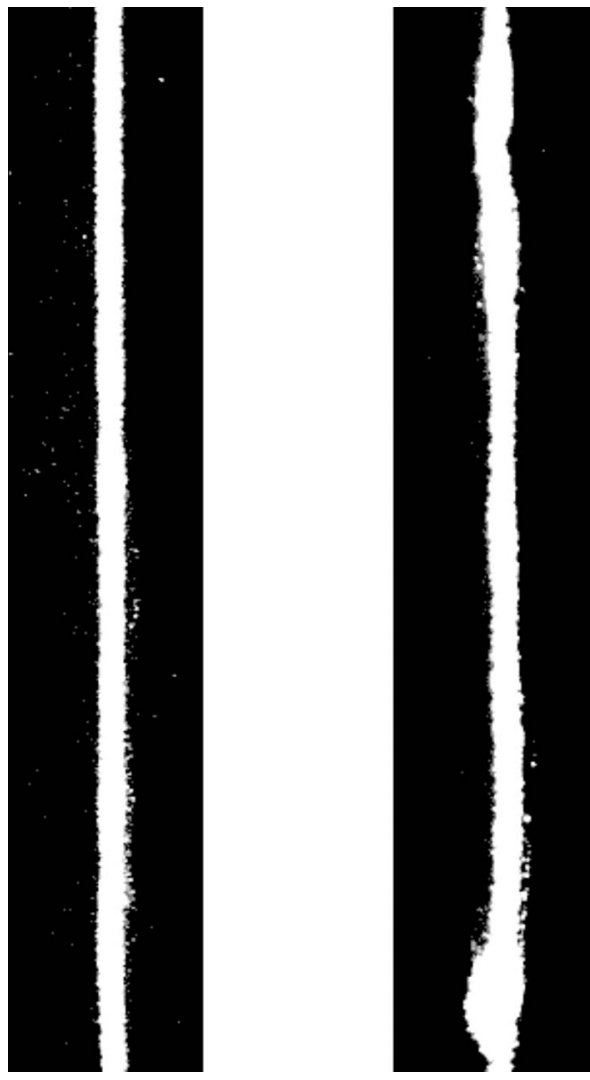

FIG. 6. Snapshots of the jet at $\phi_{0}=20 \%$ (left) and $\phi_{0}=50 \%$ (right) (enhanced online). [URL: http://dx.doi.org/10.1063/1.3276235.2]

The third equation is a Stokes equation for the pure fluid flow field $u(r)$, driven by the pressure gradient $K$,

$$
\eta_{f} \frac{1}{r} \frac{\partial}{\partial r}\left(r \frac{\partial u}{\partial r}\right)=K
$$

Finally a fourth equation imposes a zero flow rate at any horizontal cross-section of the container and accounts for a physical boundary at the bottom of the container,

$$
D_{j}^{2} V_{j}=\left(D_{c}^{2}-D_{j}^{2}\right) V_{f},
$$

where $V_{f}$ is the average fluid velocity.

Integrating and combining Eqs. (5)-(8) with the boundary conditions $v_{t}\left(D_{t} / 2\right)=0, \quad v_{j}\left(D_{j} / 2\right)=u\left(D_{j} / 2\right)$, $\eta_{f}(\partial u / \partial r)_{r=D_{j} / 2}=\eta_{j}\left(\partial v_{j} / \partial r\right)_{r=D_{j} / 2}$, and $u\left(D_{c} / 2\right)=0$ give a single nonlinear equation for the jet diameter $D_{j}$,

$$
D_{j}^{* 2}-\frac{1}{D_{j}^{* 2}}+\frac{N_{b}}{2 \eta^{*}}\left[\frac{K^{*}}{2}\left(D_{j}^{* 2}-D_{c}^{* 2}\right)-D_{j}^{* 2} \ln \frac{D_{j}^{*}}{D_{c}^{*}}\right]=0,
$$

where $D_{c}^{*}=D_{c} / D_{t}, D_{j}^{*}=D_{j} / D_{t}$, and

$$
K^{*}=\frac{K}{\left(\rho_{j}-\rho_{f}\right) g}=\frac{2+\eta^{*}-8 \ln \frac{D_{j}^{*}}{D_{c}^{*}}-2 \frac{D_{c}^{* 2}}{D_{j}^{* 2}}}{-3+\eta^{*}+4 \frac{D_{c}^{* 2}}{D_{j}^{* 2}}-\frac{D_{c}^{* 4}}{D_{j}^{* 4}}} .
$$

Equation (9) is solved numerically to find the jet diameter for each particle volume fraction. The characteristic velocity of
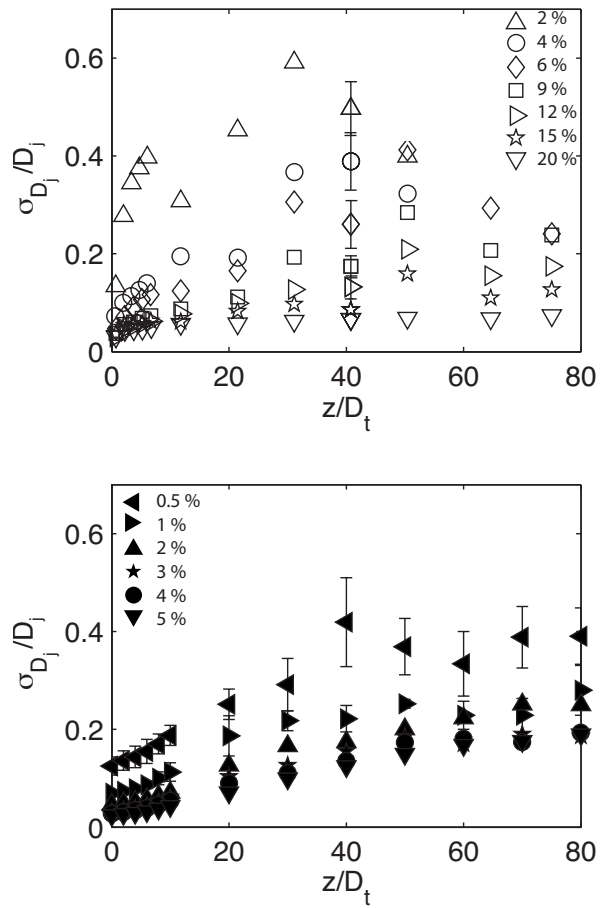

FIG. 7. Normalized standard deviation $\sigma_{D_{j}} / D_{j}$ of the jet diameter vs normalized height $z / D_{t}$ in experiments (top) and simulations (bottom) at different volume fractions $\phi_{0}$ as indicated in the graphs. In the experimental graph, error bars are only shown for $z / D_{t}=40$ for clarity. In both cases, error bars show variations between different realizations of the same experiment/ simulation and do not reflect fluctuations during the course of a given realization, which are typically much weaker.

the suspension inside the tube is $V_{t} \propto\left[\left(\rho_{j}-\rho_{f}\right) g\right] D_{t}^{2} / \eta_{j}$, and the average jet velocity is found using the mass conservation equation as $V_{j}^{*}=V_{j} / V_{t}=D_{j}^{*-2}$.

\section{GENERAL JET DYNAMICS AND GROWTH OF THE INSTABILITY}

Three qualitative jet behaviors are observed depending on volume fraction. The low volume fraction regime $\left(\phi_{0} \lesssim 20 \%\right)$ is illustrated in Fig. 5 and accompanying online movie. The front of the jet forms a mushroom-shaped cloud, which exhibits a toroidal recirculation. At the rear of this cloud, the jet presents a clear varicose instability with axisymmetric fluctuations in the local jet diameter. The pointparticle simulation is able to capture the same qualitative features. Note that the fluctuations in diameter are not accompanied by fluctuations in particulate density, which is found to remain nearly constant throughout the jet. As volume fraction is increased, the amplitude of the instability diminishes, and the jet appears to become stable for $20 \% \lessgtr \phi_{0} \lesssim 40 \%$, as seen in the left panel of Fig. 6 . At yet higher volume fractions $\left(\phi_{0} \geqslant 40 \%\right)$, large perturbations of the jet diameter are observed but without exhibiting any clear regular features (see right panel of Fig. 6). These last two regimes fall beyond the range of validity of the point-particle method, which only applies to very dilute suspensions.

In the remainder of the paper, we focus specifically on 

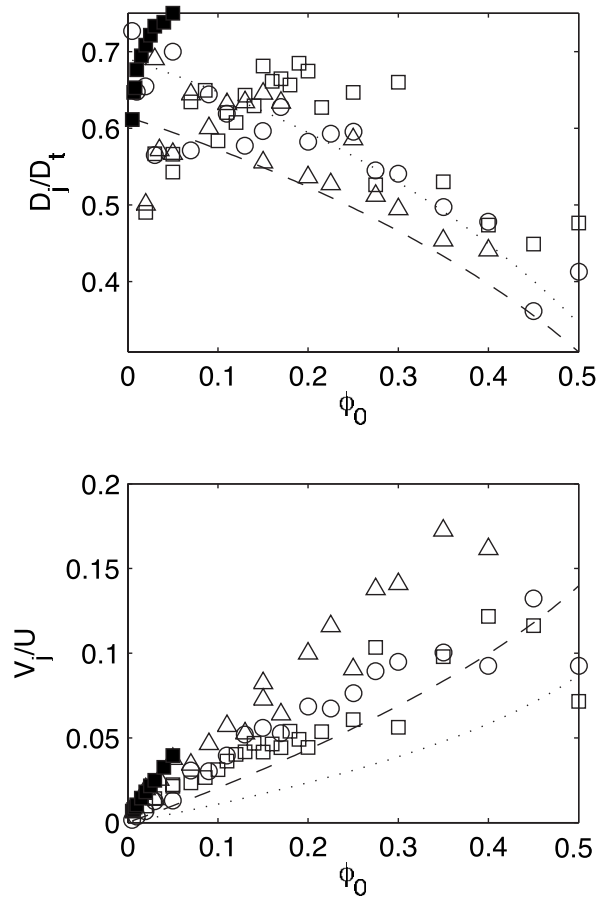

FIG. 8. Normalized mean jet diameter $D_{j} / D_{t}$ (top) and mean jet velocity $V_{j} / U$ (bottom) vs volume fraction $\phi_{0}$ from experiments: set $1(\square)$, set $2(\bigcirc)$, and set $3(\triangle)$; from simulations with $d_{p} / D_{t}=16 / 350$ corresponding to the case of sets 1 and $3(\boldsymbol{\square})$; and from the continuum model in Sec. V with $D_{t}=7 \mathrm{~mm}$, corresponding to sets 1 and 2 (dotted line), and with $D_{t}=3.3 \mathrm{~mm}$ corresponding to set 3 (dashed line). The scale $U$ used for nondimensionalization of the velocities is $U=\left(\rho_{p}-\rho_{f}\right) g D_{j}^{2} / \eta_{f}$. Error bars are not shown as they are smaller than the symbol size.

the first dilute regime, which exhibits the varicose instability. An interesting feature of the instability is that it rapidly develops in space downstream of the injection region, after which it saturates, as evidenced in the evolution of the normalized standard deviation $\sigma_{D_{j}} / D_{j}$ of the jet diameter versus normalized height $z / D_{t}$ in Fig. 7. The instability is more pronounced at low volume fractions, as shown by the increase in the growth rate with decreasing $\phi_{0}$. Note also that saturation is reached slightly closer downstream of the injection region in the experiments $\left(z / D_{t} \approx 30-40\right)$ than in the simulations $\left(z / D_{t} \approx 40-50\right)$. In the next section, we characterize this saturated regime in detail.

\section{CHARACTERIZATION OF THE SATURATED INSTABILITY}

We first start by describing average quantities such as the mean diameter and velocity of the jet and then proceed to characterizing the instability by examining quantities such as the amplitude of the diameter fluctuations and the instability wavelength.

Figure 8 (top) shows the normalized mean jet diameter $D_{j} / D_{t}$ versus volume fraction $\phi_{0}$. Two different regimes can clearly be observed. At low volume fractions, $D_{j} / D_{t}$ increases with $\phi_{0}$, whereas it decreases at high volume fractions and exhibits a maximum near $\phi_{0} \approx 10 \%-20 \%$. The decrease at high volume fractions is well captured by the continuum model. The mechanism for this decrease is the following: at high concentrations, friction is high inside the
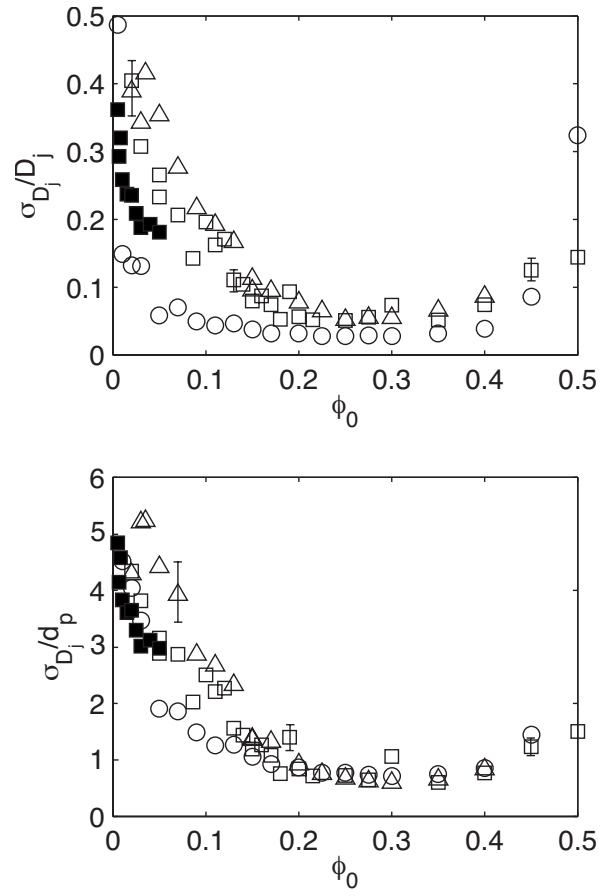

FIG. 9. Mean standard deviation $\sigma_{D_{j}}$ of the jet diameter vs volume fraction $\phi_{0}$ scaled by the mean jet diameter $D_{j}$ (top) and the particle diameter $d_{p}$ (bottom). The plots show results from experiments-set $1(\square)$, set $2(\bigcirc)$, and set $3(\triangle)$ - and from simulations with $d_{p} / D_{t}=16 / 350$ corresponding to the case of sets 1 and 3 (ם). Error bars are only shown for a few data points for indication.

injection tube owing to the higher viscosity of the suspension, which causes a stronger increase in velocity when the jet exits the tube and therefore a stronger decrease in the jet diameter by conservation of mass. The difference between the two continuum predictions results from different values of the tube-to-container diameter ratio, which influences the strength of the backflow in the pure fluid in a nontrivial manner. The initial growth of $D_{j} / D_{t}$ in the dilute regime is not predicted by the continuum model. This initial increase may be due to the perturbations of the jet diameter owing to the instability, which are strongest at low $\phi_{0}$, as we will see in Fig. 9. This trend at low volume fractions is qualitatively reproduced by the simulations, but there is a significant quantitative discrepancy. The inability of the simulations to capture quantitatively the mean diameter $D_{j}$, which is very sensitive to the exact dynamics of the suspension release out of the tube, can be attributed to the modeling of the injection process, which is not fully realistic. Note also that the pointparticle simulations cannot describe the high-concentration regimes.

Figure 8 (bottom) shows the mean jet velocity normalized by the viscous velocity scale $U=\left(\rho_{p}-\rho_{f}\right) g D_{j}^{2} / \eta_{f}$ as a function of volume fraction $\phi_{0} ; U$ corresponds to the Stokes velocity of a suspension blob of excess weight $\left(\rho_{p}-\rho_{f}\right) g$ and typical size the diameter $D_{j}$ of the jet. Two other scalings using the velocity scales $\left(\rho_{p}-\rho_{f}\right) g D_{t}^{2} / \eta_{f}$ and $\left(\rho_{p}-\rho_{f}\right) g D_{t}^{2} / \eta_{j}$ were also attempted but were less successful at gathering the data. We observe that the scaled mean velocity grows approximately linearly with $\phi_{0}$ and eventually saturates at high concentrations. This saturation can be attrib- 


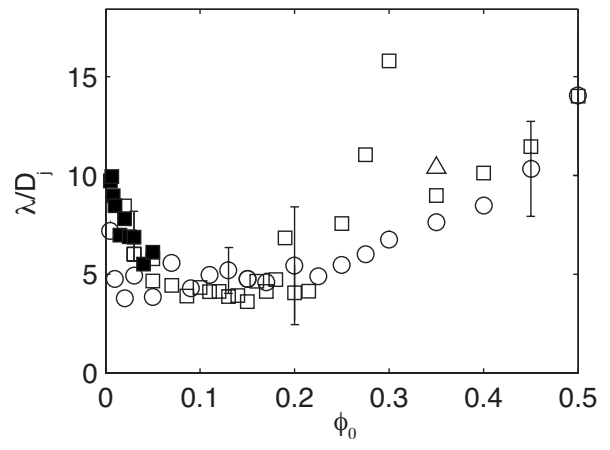

FIG. 10. Wavelength $\lambda$ of the instability normalized by the mean jet diameter $D_{j}$ vs volume fraction $\phi_{0}$ from experiments - set $1(\square)$, set $2(\bigcirc)$, and set $3(\triangle)$-and from simulations with $d_{p} / D_{t}=16 / 350$ corresponding to the case of sets 1 and $3(\square)$. Error bars are only shown for a few data points for indication.

uted to a strong increase in viscosity at high $\phi_{0}$, which decreases the flow rate through the injection tube. While both the continuum model and point-particle simulations qualitatively capture the increase in the velocity with $\phi_{0}$ at low to moderate volume fractions, quantitative differences are observed. In particular, velocities are slightly underestimated by the continuum model but are overestimated in the simulations; nevertheless, sensible agreement is obtained. In all cases, the jet velocity $V_{j}$ is found to greatly exceed the Stokes velocity of a single isolated particle (by one to two orders of magnitude depending on volume fraction), showing that the jet dynamics and the instability are driven mostly by hydrodynamic interactions.

Figure 9 shows the mean standard deviation $\sigma_{D_{j}}$ of the jet diameter, which characterizes the amplitude of the varicose instability, versus volume fraction $\phi_{0}$. Two different scalings are proposed: the top $\sigma_{D_{j}}$ is normalized by the mean diameter $D_{j}$, whereas the bottom one is normalized by the particle diameter $d_{p}$. Both scalings are valid, though we find that scaling the data with the particle diameter seems to give a better collapse. Other scalings were also attempted, including that with the injection tube outlet diameter $D_{t}$ and mean interparticle distance $\ell=\left(d_{p} / 2\right) \phi_{0}^{-1 / 3}$. While scaling the data with $D_{t}$ gave a similar collapse as with $D_{j}$, the mean interparticle distance $\ell$ was less successful at gathering the data. As previously mentioned in Sec. VI, the amplitude of the perturbations diminishes with $\phi_{0}$ to reach a minimum near $20 \% \lessgtr \phi_{0} \lesssim 40 \%$, which corresponds to approximately one particle diameter, and thus cannot be distinguished from the background noise of the discrete jet. At higher volume fractions, the perturbations are seen to increase again. In the dilute regime, good agreement is found between experiments and point-particle simulations.

The wavelength $\lambda$ of the instability, scaled by the jet diameter $D_{j}$, is shown versus $\phi_{0}$ in Fig. 10. As in the case of $\sigma_{D_{j}}$, other scalings were also attempted including $D_{t}, d_{p}$, and $\ell=\left(d_{p} / 2\right) \phi_{0}^{-1 / 3}$, but we found that $D_{j}$ results in the best collapse of the data. At low volume fractions, $\lambda$ decreases with $\phi_{0}$, reaches a flat minimum at $\approx 5 D_{j}$, and then increases again at larger concentrations. A lot of scatter is observed in this latter regime, owing to the irregularity of the perturbations at these high volume fractions. Note that the value of

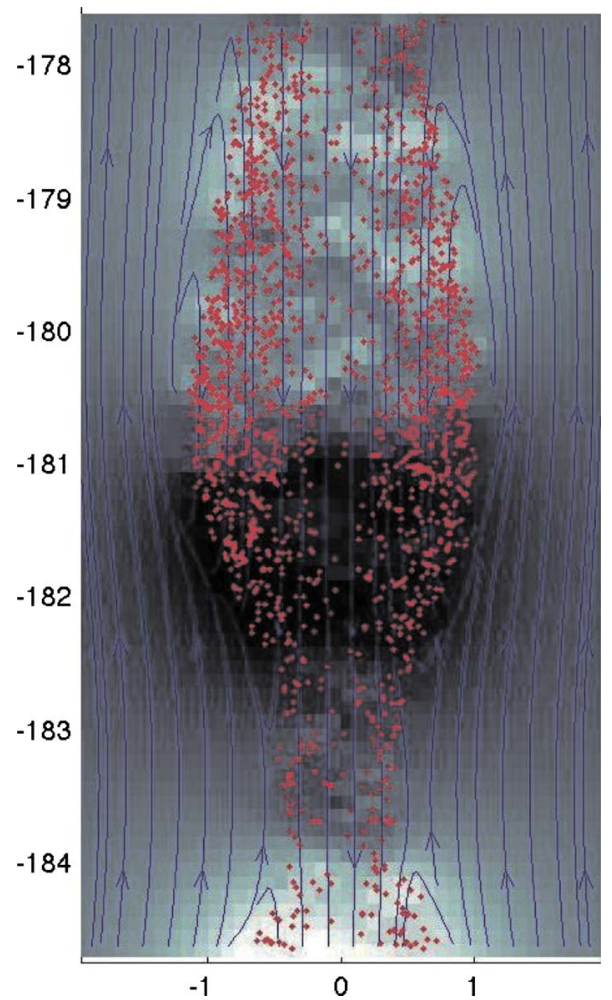

FIG. 11. (Color online) Streamlines and pressure field in a vertical plane in the reference frame moving at the mean jet velocity. High and low pressures are indicated as dark and light shades, respectively. The particles (dots) are projected onto the plane (enhanced online). [URL: http://dx.doi.org/10.1063/1.3276235.3].

this minimum is close to that of the most unstable wavelength in the Rayleigh-Plateau instability. However this is likely to be fortuitous since the mechanism for the RayleighPlateau instability is inertial in nature and involves surface tension. Once again, we obtain excellent agreement between the experimental data and the numerical results in the dilute regime.

\section{CONCLUSION}

In summary, we have investigated the dynamics of a particulate jet falling under gravity at low Reynolds number using experiments, numerical simulations, and a continuum model. Three different regimes have been evidenced depending on volume fraction. Our study has focused on the low volume fraction regime, which displays an instability in which the jet develops varicose modulation of its diameter. The instability presents a short initial growth followed by a saturated regime, which we have characterized in more detail. An important feature of the instability is that the amplitude of the perturbations decreases with increasing volume fraction and appears to vanish altogether at $\phi_{0} \approx 20 \%$. A clear wavelength selection is observed, with a dominant wavelength $\lambda$, which also decreases with $\phi_{0}$ to reach a plateau value of $\approx 5 D_{j}$ above $\phi_{0} \approx 5 \%$. A simple point-particle numerical simulation successfully captures these features of the instability. However, the mean diameter is not as well reproduced by the simulations principally because the model used for particle injection cannot account for the complex 
dynamics of the suspension release out of the tube. On the contrary, modeling the suspension as a continuum provides a good description of the mean diameter. Sensible agreement is also obtained between experiments, continuum model, and simulations for the mean jet velocity, although quantitative differences are observed.

We do not yet have a full understanding of the instability mechanism, which develops in these low-Reynolds-number particulate jets. The discrete and random nature of the particle dynamics inside the jet seems to be responsible for the development of the instability. Random fluctuations in the positions and velocities of the particles lead to small local perturbations in the jet diameter. Toroidal recirculation of the particles and of the fluid then takes place inside the bulging regions, causing the amplification of the perturbations. The jet eventually becomes a succession of suspension drops with an internal toroidal recirculation (see Fig. 11 and accompanying online movie, which shows the dynamics of this process). These drops, however, always remain attached as the particles leaking at the rear of a drop re-enter the front of the following drop. While this offers a qualitative picture of the development of the instability, a more quantitative mathematical model for the mechanism is needed.

\section{ACKNOWLEDGMENTS}

F.P. was supported by a fellowship from the French Ministère de la Recherche. D.S. gratefully acknowledges the hospitality and support of Institut Universitaire des Systèmes Thermiques Industriels (Polytech' Marseille) through a visiting professorship.

${ }^{1}$ P. G. Drazin and W. H. Reid, Hydrodynamic Stability (Cambridge University Press, Cambridge, 1981).

${ }^{2}$ J. Eggers and E. Villermaux, "Physics of liquid jets," Rep. Prog. Phys. 71, 036601 (2008).

${ }^{3}$ J. R. Lister, "Long-wavelength instability of a line plume," J. Fluid Mech. 175, 413 (1987)

${ }^{4}$ T. Funada, D. D. Joseph, and S. Yamashita, "Stability of a liquid jet into incompressible gases and liquids," Int. J. Multiphase Flow 30, 1279 (2004).

${ }^{5}$ M. Nicolas, "Experimental study of gravity-driven dense suspension jets," Phys. Fluids 14, 3570 (2002).

${ }^{6}$ J. M. Nitsche and G. K. Batchelor, "Break-up of a falling cloud containing dispersed particles," J. Fluid Mech. 340, 161 (1997).

${ }^{7}$ G. Machu, W. Meile, L. C. Nitsche, and U. Schaflinger, "Coalescence, torus formation and break-up of sedimenting clouds: Experiments and computer simulations," J. Fluid Mech. 447, 299 (2001).

${ }^{8}$ B. Metzger, M. Nicolas, and É. Guazzelli, "Falling clouds of particles in viscous fluids," J. Fluid Mech. 580, 283 (2007).

${ }^{9}$ I. M. Krieger, "Rheology of monodisperse lattices," Adv. Colloid Interface Sci. 3, 111 (1972). 